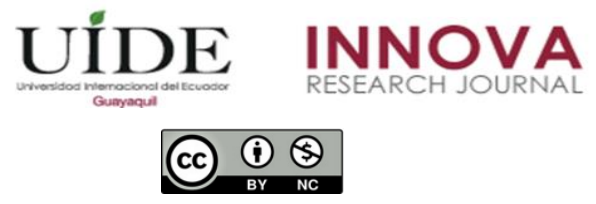

INNOVA Research Journal, ISSN 2477-9024

(Septiembre-Diciembre 2020). Vol. 5, No.3 pp. 78-92

DOI: https://doi.org/10.33890/innova.v5.n3.2020.1402

URL: http://revistas.uide.edu.ec/index.php/innova/index

Correo: innova@uide.edu.ec

\title{
La Academia de Diseño en el escenario de la Innovación Tecnológica
}

\section{The Design Academy on the scene of Technological Innovation}

Laura Teresa Gómez-Vera

https://orcid.org/0000-0002-4191-4293

Universidad Autónoma del Estado de México, México

Autor para correspondencia: lagov13@gmail.com

Fecha de recepción: 23 de abril del 2020 - Fecha de aceptación: 10 de agosto del 2020

\section{Resumen}

En este artículo se expone, desde una perspectiva teórica, la gestión integral del diseño para hacer frente a la práctica pedagógica por objetivos e incitar la innovación tecnológica en el desarrollo de proyectos que deriven en bienes y servicios innovadores. Particularmente, en los escenarios académicos mexicanos es conveniente trabajar en torno a las funciones del "Modelo de Gestión de la Tecnología" que planteó, desde el 2012, la Fundación del Premio Nacional de Tecnología e Innovación. Se recurrió al análisis cualitativo de explicaciones teóricas con base en los principios metodológicos descriptivos para exponer la analogía entre la organización de la academia, la intervención pedagógica y la deducción cognitiva en el ámbito de la "planeación, vigilancia, habilitación, protección e implantación tecnológica" (FPNTI, 2016). La interrelación pedagógica que se provoca con la dirección integral de proyectos de diseño es el resultado que se espera en la profesionalización de este campo de estudio.

Palabras Claves: diseño; proyectos; gestión; pedagogía; objetivo

\begin{abstract}
This article presents, from a theoretical perspective, the integral management of design to address pedagogical practice by objectives and to encourage technological innovation in the development of projects that lead to innovative goods and services. Particularly, in Mexican academic scene it is convenient to work around the functions of the "Technology Management Model" that the National Technology and Innovation Award Foundation proposed since 2012. The qualitative analysis of theoretical explanations based on descriptive methodological principles was used to explain the analogy between the organization of the academy, pedagogical intervention and cognitive deduction in the field of "planning, monitoring, habilitation, protection and technological implementation". (FPNTI, 2016). The pedagogical interaction that is provoked with the integral management of design projects is the result that is expected in the professionalization of this field of study.
\end{abstract}

Key Words: design; project; management; pedagogy; objectives 


\section{Introducción}

La práctica pedagógica en la disciplina del diseño enfrenta desafíos que, si bien se atribuyen a la metodología académica a la que se recurre, se ve que la implantación de los objetos que se generan está fuera de la realidad en la esfera social, confundiendo así su identidad en el ejercicio profesional. Esto es una consecuencia que la formación especializada enfrenta de cara a la mundialización, y que ha provocado, en la opinión de Díaz (2013) "un modelo hegemónico basado en estándares y competencias” (p. 178).

La academia de diseño siempre ha mostrado un interés por cimentar un razonamiento crítico que sostenga a esta disciplina como una inteligencia para el equilibrio social, lo cual es un factor decisivo para que el proceso de innovación "se cristalice y se radique en un paradigma que promueva el conocimiento complejo" (Morín, 1999, p. 11), al tiempo de abordar la inadecuación que deviene del problema universal de la educación, que versa sobre el contexto, lo global y lo multidimensional. (Morín, 1999). Lo central en ese debate se dirige hacia la extensión de la práctica pedagógica con base en estrategias novedosas para optimizar su intervención.

Aun cuando la atención que se otorgue a los principios del aprendizaje, -tales como los que señalan Campos, Bolbarán, Bustos y González (2014), relativos a la gerencia de proyectos, al mejoramiento de programas educativos y a la intervención cognitiva de todos los actores académicos, -sea determinante en la calidad del proceso, hay que resaltar que hay otras funciones externas en donde las instituciones y sus educandos pueden potenciar el proceso creativo y asegurar su intervención en proyectos para el desarrollo social.

No obstante, la dinámica de la innovación se haya desplegado en algunas entidades industriales mexicanas que buscan obtener mayor competitividad en su gremio, se observa que todavía en la práctica académica para la formación de profesionales del campo del diseño, ésta es inadvertida. El currículo de los programas educativos que concurren en la esfera de la gestión del diseño se orienta principalmente al conglomerado de conocimientos relativos al temas de emprendimiento, prescindiendo de otras nociones que procuran el conjunto de la innovación.

En esa perspectiva es propicio reflexionar y abordar el proceso de enseñanzaaprendizaje en el área del diseño bajo los preceptos que determina la innovación tecnológica, que hace que los procesos de desarrollo se conduzcan de tal manera para que las propuestas puedan escalar hacia la transferencia tecnológica.

\section{Dimensión pedagógica en la gestión de proyectos integrales de diseño}

Los sistemas flexibles de enseñanza-aprendizaje están previstos para impulsar mejores prácticas y redes de colaboración que son necesarias en la realización de proyectos en los que prevalece la importancia de recuperar la realidad epistémica a través del ejercicio de aprendizaje del educando, al tiempo de que éste se distinga como aquel que origina el conocimiento que es necesario para caracterizar una realidad. (Hernández, 2008). Esta dimensión incita al docente a afrontar los inconvenientes que puedan deprimir el interés del estudiante para su aprendizaje 
integral, así como abrir nuevas rutas de conocimiento que se construyan con base en la experiencia. (Álvarez, 2011).

Experimentar prácticas pedagógicas alternativas a las tradicionales tiene sentido para gestionar proyectos innovadores de diseño bajo ese cometido. Al tiempo de atender las variables por demás conocidas en los argumentos formales, funcionales y de manufactura en el campo de la creatividad plástica y material, la innovación ocurre en el entramado de un pensamiento integral para lograr que aquellos quehaceres que afectan el devenir de ciertas comunidades y regiones sean resueltos de forma multidimensional, provocando ciertas circunstancias que revelen mejoras en la conducta y en la forma de proceder de los involucrados.

En un sentido amplio, la innovación es más una consecuencia que una acción. Es integrar todos los escenarios que sea posible para hacer frente a las carencias que están presentes en los entornos local, regional y global al perseguir un progreso armónico. Esta práctica se reconoce por instancias internacionales que coinciden en que la innovación sucede hasta el momento en que las invenciones sean implementadas o comercializadas para formar parte en algún modelo de negocio (IMNC-2007a). Tal como se explica en el Manual de Frascati, emitido por la Organización para la Cooperación y el Desarrollo Económicos (2003), se trata de desarrollos basados en metodologías que domina la ciencia y la técnica, o bien en esquemas que favorezcan el quehacer de la organización y la comercialización de productos y servicios.

Cuando se busca originar una intervención de diseño, no siempre éste es coincidente en ambientes de trabajo híbridos, por lo que se sugiere, en un inicio, aproximarse desde un discernimiento conceptual. Como un ejercicio, se expone los argumentos siguientes:

Diseño, cultura y sociedad. El diseño, como medio de comunicación social y cultural es una manera de distinguir los significados que los objetos provocan en los individuos, en los espacios que utiliza y en las experiencias que adquiere en su actuar cotidiano; y que, mediante diversos escenarios en su apropiación, revelan el valor social y cultural que éstos representan. Los artefactos-objetos, así como otras expresiones del diseño, son medios de interacción del individuo con su medio, lo cual es una franca oportunidad para atender, prioritariamente, asuntos de interés masivo en temas particulares que atañen a la salud física y mental de los individuos u otros organismos vivos, a la equidad de género, al mejoramiento ambiental, a la eficiencia del hábitat y de su entorno, a la movilidad, etcétera. Asimismo, da cabida a otras propuestas que beneficien a la miscelánea artesanal, al abasto alimenticio, o la recreación, entre muchos otros temas de particular importancia en el equilibrio de las coyunturas sociales.

Diseño y tecnología. La coyuntura entre el diseño y la tecnología se deriva conceptualmente con el saber optar por recursos tangibles e intangibles que, en conjunto, revistan mayor potencial a las soluciones de diseño de forma ordenada y metódica. (IMNC, 2007b, p. 3). En este escenario, el diseño se desenvuelve como un mecanismo de gestión. Así, se puede enfrentar la sinergia que se presenta entre especialistas de diversos ramos a fin de articular una visión epistemológica; su pertinencia radica en resolver las dimensiones técnicas, de calidad y de manufactura de productos y procesos a cualquier escala. 
Diseño y mercado. Los argumentos que hacen factible la transferencia de productos de diseño, como dispositivos para engranar los requerimientos internos de una organización con otros de carácter global, observa aquellas condicionantes que hilvanan las prioridades de los conglomerados sociales con las oportunidades empresariales bajo el enfoque de negocio que, a su vez, hagan evidente la competitividad de algún sector para fomentar comunidades socioproductivas que sustenten un equilibrio económico, social y ambiental.

\section{Abordaje académico en la dirección de proyectos de innovación}

Las buenas prácticas se identifican a partir de la delimitación de actividades dispuestas para proyectos de ejecución temporal o progresiva, que se encaminan para asociar el valor de las soluciones tecnológicas (IMNC, 2007b). También ofrecen información teórica y técnica que fundamenta otras intervenciones, o bien que resultan en productos materiales e inmateriales determinados. Los proyectos de innovación han de distinguirse según su origen: ya sea, derivados de una oportunidad de mercado, por una demanda tecnológica, o bien por requerimientos de uso y función (manipulación, confiabilidad, seguridad), o estructurales y técnicos (producción y suministro de materiales) de los artefactos sujetos a mejoras con base en las tendencias tecnológicas globales.

La dirección de los proyectos de innovación debe lograr el aprendizaje significativo entre los integrantes de un equipo de diseño, haciendo uso de experiencias diversas que respondan a las funciones de gerencia, de intercambio y de transacción para cumplir con los objetivos y requisitos que corresponda a cada caso; además de proporcionar la retroalimentación precisa a fin de optimizar sus resultados conforme a las especificaciones creativas y de transferencia oportunamente especificadas. En el tipo de intervenciones por incidentes o por problemas es altamente formativo el análisis de situaciones sobre una realidad, lo cual se puede llevar a cabo mediante técnicas de inmersión, experimentación e investigación, a fin de proponer soluciones en campos tecnológicos; en cambio, en las intervenciones de asociación de los enfoques teóricos del diseño, mismos que podemos apreciar, por ejemplo, en la conceptualización del llamado diseño colaborativo, diseño universal, emocional o de experiencias son las coincidencias de un proceso creativo en una situación determinada las que dan lugar a ampliar la capacidad cognitiva a fin de establecer y compartir nuevas representaciones hipotéticas.

Considerando que los proyectos de innovación no son lineales, es menester que la intervención pedagógica sea flexible. Esta precisión induce a desdoblar manejos didácticos que privilegien el trabajo colaborativo, indistinto en cada faceta, para abrir mayores conexiones dinámicas, integrales y críticas entre estudiantes, docentes y otros asociados. Se entiende que sea posible que el grupo que inicia un proyecto determinado no sea el mismo que participe en todas y cada una sus fases.

Claro está que el esquema de aprendizaje colaborativo tiene varias asimetrías; entre ellas la falta de definición de variables e indicadores para el seguimiento y la evaluación de los proyectos, que va más allá de contenidos de aprendizaje y de rúbricas predefinidas. Se trata de que todos los participantes asuman una postura en donde la apreciación y la pertinencia de las soluciones sea un proceso continuo para construir un aprendizaje significativo. Es importante que la estructura jerárquica de los grupos sea horizontal, siempre que se distingan las categorías 
de docentes y discentes, según las competencias que exija el óptimo rendimiento de cada proyecto. Para esto se sugiere distinguir, por ejemplo, los ámbitos de desarrollo siguientes:

Dominio cognitivo: saber conducir el análisis de las informaciones que son vigentes y relevantes en el campo conceptual, técnico e histórico para contextualizar y distribuir el conocimiento, así como comparar los contenidos de grupos de datos, antecedentes, hechos, etcétera.

Dominio psicomotor: saber gestionar interfaces y resolver problemas para incidir en procesos de creación de objetos, procesos y métodos bajo estándares de calidad. Conducir los proyectos en situaciones de aprendizaje de diversa índole es impulsar un dominio en estrecha relación con las habilidades de negociación y liderazgo para que los procesos alcancen la transferencia tecnológica, bajo el pensamiento de diseño.

Dominio afectivo: saber conducir los intereses de los grupos y fomentar la tolerancia y el respeto hacia el entendimiento común a fin de intervenir en la gestión congruente de un sistema o subsistema. Cuando los actores muestran empatía en entornos sociales diferenciados, les permite influir en esos escenarios con propuestas y argumentos específicos.

\section{Gestión del diseño en y para incitar la innovación tecnológica}

La gestión del diseño, con motivo de hacer frente a una nueva cultura de enseñanza aprendizaje, es la base en la que se sustenta la efectividad de la estrategia de diferenciación de valor y de la dinámica de excelencia competitiva. La tarea a la que se enfrentan los actores académicos es crear escenarios educativos destacados, cuyas competencias distingan al gremio profesional del diseñador. Con base en las funciones del modelo previamente mencionado, a continuación, se expone la intervención pedagógica en relación con la cultura organizacional y sus características, para así presentar la deducción cognitiva de cada argumento.

\section{Vigilancia tecnológica}

El análisis que deriva en el uso eficiente de la información técnica que se genera en el mundo de la innovación es una tarea por demás compleja, en la medida que la publicación sistemática de esas informaciones tiene un crecimiento exponencial, además que la dificultad de su estudio radica en que cualquier tipo o clase de innovación está vinculada con una serie de variables sociales, económicas, técnicas o ambientales interrelacionadas.

Esta función se entiende como un conocimiento que fortalece la coordinación de los proyectos al observar el estado de la técnica de un protocolo determinado; particularmente para observar la movilidad de las tendencias mundiales de investigación y desarrollo tecnológico. Implica definir el alcance, en años de estudios retrospectivos, y determinar las fuentes de información más adecuadas para dar respuesta a los límites previstos. Este ejercicio debe, a su vez, obedecer a un método preciso que aporte con veracidad el comportamiento sobre el mercado de productos y servicios análogos al objeto de estudio que estén disponibles en el entorno local, nacional e internacional a fin de estudiar con precisión sus características formales, funcionales y técnicas. 


\section{Tabla 1.}

\section{Intervención pedagógica en la función de vigilancia tecnológica}

\begin{tabular}{ll}
\hline Cultura Organizacional & Características \\
\hline $\begin{array}{l}\text { a. Construir y mantener una red de fuentes de } \\
\text { información tecnológica como factor clave en el } \\
\text { proceso de diseño y de manufactura. }\end{array}$ & $\begin{array}{l}\text { Saber registrar la información acerca de las capacidades, los } \\
\text { antecedentes, los perfeccionamientos y los proyectos y sus } \\
\text { resultados, así como discernir sobre las características } \\
\text { científicas y técnicas. }\end{array}$ \\
$\begin{array}{l}\text { b. Monitorear las informaciones especializadas } \\
\text { distinguiendo el carácter del objeto de estudio. }\end{array}$ & $\begin{array}{l}\text { Saber analizar y sistematizar argumentos con base en } \\
\text { metodologías de prospectiva tecnológica. }\end{array}$ \\
$\begin{array}{l}\text { c. Generar un insumo permanente de información } \\
\text { para apoyar la toma de decisiones en torno a las } \\
\text { condicionantes creativas y técnicas en el marco de } \\
\text { las innovaciones. }\end{array}$ & $\begin{array}{l}\text { Saber aportar una serie de testimonios con base en una } \\
\text { amplia gama situaciones tecnológicas. }\end{array}$ \\
$\begin{array}{l}\text { d. Extender la difusión de la información entre las } \\
\text { entidades interesadas. }\end{array}$ & $\begin{array}{l}\text { Saber expresar las condiciones y necesidades de cambio en } \\
\text { el entorno del objeto de estudio. }\end{array}$
\end{tabular}

Fuente: Elaboración propia

\section{Deducción cognitiva}

a. Compendio de fuentes de información procesadas y otras secundarias. Apropiarse crítica y creativamente de herramientas tecnológicas con base en una dimensión axiológica para discernir en las esferas cognitiva, instrumental y comunicativa (OEI, 2014, s/p), entendiendo que los medios de acceso abierto son el primer acercamiento al conocimiento (revistas científicas y técnicas, libros, bases de datos de patentes y otras figuras de propiedad industrial). Adicionalmente, saber que el propio sector empresarial y los proveedores comerciales disponen de sus propios expedientes que revelan el estado del arte en un campo y de un producto determinado.

b. Mapeo de trayectorias tecnológicas. Entender sobre el suministro y la diferenciación de los contenidos técnicos, históricos y legales que se desprenden del conocimiento científico, del desarrollo tecnológico y del Know How.

c. Cartera de tendencias tecnológicas. Derivado del análisis de una averiguación previa, es posible llevar a cabo el proceso que se conoce como inteligencia competitiva a fin de detectar tendencias, analizar reacciones, opiniones y estrategias de mercado, socios potenciales, hacer comparativas y valoraciones, o emitir señales del entorno sobre usuarios, competidores, proveedores y regulaciones, entre otras informaciones.

d. Registro y reporte de la gestión de la información. Discernir sobre la suficiencia de infraestructura tecnológica a fin de garantizar la disposición y la consulta de las informaciones, mismas que puede comprender observatorios tecnológicos, redes de monitoreo y sistemas de clasificación y de indicadores, por mencionar algunos ejemplos. 


\section{Planeación tecnológica}

El Plan Tecnológico tiene el propósito de apoyar el trabajo por proyectos y su revisión permanente. En la Norma Mexicana Sistema de Gestión de la Tecnología-NMX-GT-003, la planeación tecnológica se define como un proceso para anticipar el comportamiento tecnológico que circunda a las organizaciones para actuar en consecuencia. (IMNC, 2007a). Por su parte, Barjau (2006), añade que se trata de una función que induce al análisis, a la definición de objetivos tecnológicos y a la organización de los recursos económicos, materiales, humanos y tecnológicos.

La planeación tecnológica se registra como una acción que prepara lo necesario en los proyectos que seleccionan los organismos para dar la oportunidad de cooperar con nuevos socios, clientes, proveedores u otros expertos. Esta fase induce a revisar las capacidades propias con el propósito de progresar y detectar aquellas divergencias que se presenten entre los productos diseñados y las necesidades de los usuarios, así como reducir riesgos en la medida de detectar amenazas derivadas de patentes, reglamentos, alianzas o nuevas inversiones.

La posición estratégica de la organización en torno a un proyecto de diseño es determinante para el desarrollo de un plan de acción que vaya de acuerdo con el potencial de los recursos humanos, materiales y la infraestructura de que se disponga y que, a su vez, garantice una cartera de proyectos estable y la mejor sinergia entre los interesados de la comunidad académica. Si esto es favorable, será la fuente generadora del cambio para alentar un proceso organizacional estructurado y compartido.

\section{Tabla 2.}

\section{Intervención pedagógica en la función de planeación tecnológica}

\section{Cultura Organizacional}

a. Monitorear las carencias y problemas no resueltos que provocan algún desequilibrio social en un contexto específico, que se puedan atender bajo el enfoque del diseño.

b. Formular un marco estratégico con oportunidad de atender una condición coyuntural bajo los estándares del diseño con proyectos específicos.

c. Determinar los requerimientos humanos que sean precisos para realizar cada proyecto y cada una de sus fases.

\section{Características}

Saber reconocer las oportunidades de intervención, derivando de ello la viabilidad de enlace interinstitucional.

Saber definir las líneas estratégicas que den respuesta a las necesidades y a los retos tecnológicos, y saber conducir las oportunidades con potencial de innovación.

Saber discernir sobre las competencias disciplinares y las cualidades conductuales de los sujetos interesados.

Fuente: Elaboración propia

\section{Deducción cognitiva:}

a. Identificación de oportunidades de nichos de innovación en sectores estratégicos. Comprender y exponer los escenarios de entidades sociales y empresariales a fin de delimitar tanto los factores que determinan su competitividad como el comportamiento de mercados 
análogos, a fin de exponer las oportunidades que las alianzas reconozcan. Se busca señalar con claridad la posición estratégica de la organización y sus objetivos.

b. Definición de la cartera de proyectos y plan de seguimiento. Definir el contenido tecnológico que pretende conjugar oportunidades técnicas con necesidades para introducir o modificar sistemas, objetos o servicios con fines de comercialización. Por lo general estos paquetes contienen tecnología de producto, de proceso, de equipo y de operación con potencial de presentar novedad, actividad inventiva y aplicación industrial. Características señaladas en el artículo 12 de la Ley de la Propiedad Industrial (Cámara de Diputados del H. Congreso de la Unión, 2018).

c. Identificación de grupo de asesores e interesados. Distinguir, con base en las competencias académicas y profesionales requeridas para intervenir en un proyecto, a los interesados como aquellas personas, grupos e instituciones idóneas. En tanto que la gestión participativa es un factor de éxito, los interesados comparten la responsabilidad de llevar a cabo el desarrollo y el seguimiento del proyecto hasta el logro de sus objetivos.

\section{Habilitación tecnológica}

La habilitación tecnológica significa trabajar en congruencia con las dinámicas del exterior para resolver las necesidades tecnológicas que presenten las iniciativas de los portafolios (FPNT, 2016). A fin de asegurar la calidad de los protocolos se involucran los procesos de gerencia para apoyar los asuntos que tienen que ver con el trabajo en equipo, con la trascendencia de las iniciativas, con el establecimiento y cumplimiento de los periodos establecidos, con el coste, con los procesos de interacción, etc. Esta labor sugiere, además, determinar las modalidades para la explotación comercial de las innovaciones.

Tabla 3.

Intervención pedagógica en la función de habilitación tecnológica

\begin{tabular}{ll}
\hline Cultura Organizacional & Características \\
\hline $\begin{array}{l}\text { a. Determinar los requerimientos } \\
\text { técnicos y materiales para ejecutar cada } \\
\text { proyecto haciendo una diferenciación en } \\
\text { cada una de sus fases. }\end{array}$ & $\begin{array}{l}\text { Saber discernir sobre las condiciones humanas, técnicas y materiales } \\
\text { cada proyecto. }\end{array}$ \\
$\begin{array}{ll}\text { b. Priorizar el desarrollo de la } \\
\text { investigación y el desarrollo } \\
\text { tecnológico. }\end{array}$ & $\begin{array}{l}\text { Saber dirigir la investigación y el desarrollo tecnológico con fines de } \\
\text { escalamiento y transferencia tecnológica. }\end{array}$ \\
$\begin{array}{l}\text { c. Conducir la transferencia de la } \\
\text { tecnología. }\end{array}$ & $\begin{array}{l}\text { Saber generar estrategias de negocio y definir mecanismos de } \\
\text { escalamiento comercial de activos tangibles e intangibles. }\end{array}$ \\
$\begin{array}{l}\text { d. Gestión de la cartera de proyectos } \\
\text { tecnológicos. }\end{array}$ & Saber compartir asistencia técnica y consultoría tecnológica. \\
e. Gestión de recursos tecnológicos. & $\begin{array}{l}\text { Saber proyectar la habilitación tecnológica de los participantes a fin de } \\
\text { conducir la negociación del proyecto con las entidades vinculantes. }\end{array}$
\end{tabular}




\begin{tabular}{ll}
\hline Cultura Organizacional & Características \\
\hline f. Gestión de recursos financieros. & $\begin{array}{l}\text { Saber registrar potenciales empresas patrocinadoras, fondos } \\
\text { concursables u otros acervos que operen con capital de riesgo. }\end{array}$ \\
g. Gestión del conocimiento. & $\begin{array}{l}\text { Saber elaborar programas de consultoría en materia de diseño y } \\
\text { desarrollo de tecnología. }\end{array}$ \\
\hline
\end{tabular}

Fuente: Elaboración propia

\section{Deducción cognitiva:}

a. Adquisición de tecnología. Con base en el estudio de mercados y clientes, conocer los términos para llevar a cabo la compra, las licencias y las alianzas particulares que sean pertinentes para la ejecución de los proyectos.

b. Asimilación de infraestructura tecnológica. Con base en estudios de tendencia de diseño y de patrones de producción-consumo, saber tomar las decisiones técnicas y de negocio que sean favorables para la implantación y comercialización de la tecnología.

c. Otorgamiento y adquisición de licencias y elaboración de acuerdos. Con base en estudios de competitividad es posible originar modelos de negociación con entidades vinculantes de carácter sectorial, a fin de que el conocimiento de los participantes aporte los términos idóneos de los instrumentos legales que tengan lugar en concordancia con la normatividad vigente que aplique a cada caso. Identificar la posición del producto o servicio según las condiciones del mercado. Saber gestionar modelos para determinar el valor de las innovaciones. Realizar alianzas con otras entidades y diseñar los instrumentos y tipo de acuerdos que respondan a las necesidades de los proyectos y de los interesados.

d. Diseño de métodos de gestión de proyectos. Esta deducción, reviste saber proveer políticas y metodologías para realizar los proyectos, ajustándose a las condiciones técnicas, operativas y legales de las instancias implicadas. Se debe partir del reconocimiento del origen de los proyectos, ya que de ello depende la intervención metodológica, sea que se trate de la demanda empresarial o impulsados por la investigación básica, aplicada y de desarrollo tecnológico, además de las necesidades específicas en los ámbitos local, estatal, regional, nacional e internacional.

e. Competencias y habilitación tecnológica de los interesados. Saber organizar los contenidos que son necesarios para el desarrollo de los portafolios y actuar en consecuencia de los planes y modelos previstos a fin de conducir las competencias hacia el aprovechamiento de los recursos humanos, materiales y económicos. Esto con el cometido de solucionar todo aquello que intervenga en la inserción de cambios tecnológicos en las empresas que buscan una mejor posición competitiva. (FPNTI, 2016).

\section{f. Disponibilidad de patrocinadores y recursos financieros. La transferencia y} comercialización de los proyectos que están inmersos en un entorno competitivo y global de cambios tecnológicos acelerados dependen de posibilidades reales de financiación. Además de un ejercicio racional, con eficiencia y transparencia de la aplicación de los recursos que garantice la optimización de estos. Buscar incentivos y partidas presupuestales (convocatorias, recursos 
públicos, etcétera) e identificar capital de inversión y gestionar fondos para la transferencia de las innovaciones.

g. Registro de contenidos, procedimientos y lecciones aprendidas. Desarrollar contenidos específicos para ampliar las capacidades tecnológicas mediante procesos de consultoría orientados, según sea el caso, a la demanda local, nacional o internacional. Asimismo, saber conformar los registros que sean necesarios para transmitir el conocimiento adquirido. Dominar el registro de información con respecto a las empresas con interés de vincularse para los efectos de comercialización y explotación de los productos y servicios que se generen.

\section{Protección de la tecnología}

La protección tecnológica se refiere, desde la visión de los inventores, a un reconocimiento para recompensar la inversión de recursos creativos, además del tiempo empleado en la generación de obras o producciones industriales. Y desde el enfoque corporativo, se considera como un recurso que limita la competencia alevosa que provoca la alteración de productos, procesos y servicios originales.

El sistema internacional de protección intelectual comprende dos ramas: Propiedad Industrial y Derechos de Autor. Los títulos que otorga el primero se refiere a las invenciones tecnológicas de aplicación industrial y a inferencias publicitarias que se utilizan para reconocer objetos y bienes tangibles, principalmente de base tecnológica. Tiene beneficios en cuanto a exclusividad, temporalidad y territorialidad. Los Derechos de Autor son concedidos por el desarrollo de obras de la literatura o producciones artísticas.

Los derechos de propiedad intelectual estimulan a las empresas y a otras organizaciones a mejorar las formas de comercialización que utilizan en sus actividades productivas. Evitan la invasión de los derechos adquiridos, lo que fortalece y regula el mercado interno; permiten licenciar los derechos tecnológicos; facilita el intercambio comercial a través de alianzas estratégicas; y es también un factor para la negociación en tratados comerciales de los países.

Las competencias que son necesarias en este tema deben garantizar una pericia en aspectos normativos y legales que permita definir una estrategia de protección y explotación del conocimiento con base en los intereses comerciales de las organizaciones involucradas y de acuerdo con las dinámicas tecnológicas en entornos regionales de atracción.

Tabla 4.

Intervención pedagógica en la función de protección de la tecnología

\begin{tabular}{ll}
\hline Cultura organizacional & Características \\
\hline $\begin{array}{l}\text { a. Facilitar la administración de la } \\
\text { propiedad industrial. }\end{array}$ & $\begin{array}{l}\text { Saber caracterizar las innovaciones para su registro según las figuras de } \\
\text { propiedad intelectual que determina la Ley de la Propiedad Industrial. }\end{array}$ \\
$\begin{array}{l}\text { b. Facilitar la administración de } \\
\text { derechos de autor. }\end{array}$ & $\begin{array}{l}\text { Saber caracterizar las innovaciones para su registro según las figuras de } \\
\text { derechos de autor que determina la Ley de la Propiedad Intelectual. }\end{array}$
\end{tabular}




\begin{tabular}{ll}
\hline Cultura organizacional & Características \\
\hline $\begin{array}{l}\text { c. Gestión de patentes y otras figuras de } \\
\text { propiedad intelectual. }\end{array}$ & $\begin{array}{l}\text { Saber ejecutar los alcances, ratificaciones y negociaciones de los títulos } \\
\text { de propiedad intelectual. }\end{array}$ \\
d. Difusión de la protección intelectual. & $\begin{array}{l}\text { Saber comunicar los resultados del registro de solicitudes y obtención } \\
\text { de registros de propiedad industrial e intelectual. }\end{array}$ \\
$\begin{array}{l}\text { e. Ampliar y atender lo referente al } \\
\text { acervo tecnológico. }\end{array}$ & $\begin{array}{l}\text { Saber explotar los recursos intangibles, y levar a cabo el licenciamiento } \\
\text { de patentes y demás títulos de propiedad intelectual. }\end{array}$ \\
\hline
\end{tabular}

Fuente: elaboración propia

\section{Deducción cognitiva:}

a. Estrategias para la protección del patrimonio tecnológico. Saber advertir las ventajas competitivas que ofrece el sistema oficial de protección de la tecnología y conocer las posiciones del entorno comercial y empresarial (IMNC, 2007b). Gestionar las innovaciones siguiendo las especificaciones de los registros que corresponda, según lo que señala la Ley de Propiedad Industrial. El proceso inicia distinguiendo las características de la innovación, la novedad y la aplicación industrial del objeto o proceso desarrollado, de manera que su evaluación se basa en el potencial comercial que se justifique para su registro a nivel nacional o en otros países en donde se decida comercializar el producto, el proceso o el método.

b. Estrategias para la protección del derecho de autor. Los Derechos de Autor son concedidos por el desarrollo de obras literarias o artísticas. El proceso inicia distinguiendo los derechos que corresponden a cada una de los creadores y entidades que generaron la obra a través de sus contribuciones intelectuales. Su gestión requiere distinguir la tipología de derechos de autor conforme a la normativa vigente y los procedimientos para su registro.

c. Registro y seguimiento de las solicitudes en el contexto del país y el extranjero. La gestión de títulos de propiedad intelectual incluye elaborar los informes que requiere el proceso de registro y el proceso para mantener vigente los derechos, así como verificar que se realicen en tiempo y forma los pagos que corresponda a los periodos señalados en los títulos adquiridos, y conforme a lo que determina la ley de la propiedad industrial.

d. Difusión interna y externa de resultados de obtención de registros y su explotación. La difusión oportuna de iniciativas de vinculación y sus resultados contribuyen a mejorar las condiciones tecnológicas del país, en tanto la apertura de programas que acerquen a la comunidad académica a las prácticas que formen parte de las estrategias tecnológicas de las organizaciones.

e. Contratos y acuerdos de licenciamiento y explotación de patentes. Regular la venta o la concesión de las patentes u otro título de propiedad intelectual, así como vigilar la autorización de las licencias para su explotación por parte de terceros. Saber gestionar los ingresos y egresos que se reporten por la explotación de las licencias otorgadas y recibidas. 


\section{Implantación tecnológica}

Esta función incluye todas aquellas prácticas que van desde el proceso de creación de objetos y servicios tecnológicos hasta su introducción en el sector comercial de interés. (FPNTI, 2016). Incluye la evaluación del proyecto y de los beneficios derivados del proceso.

Ya sea que se trate de proyectos impulsados por la investigación (technology push), o por aquellos impulsados por la demanda empresarial (market pull), las organizaciones deben introducir en el equipo y documentar cada una de las fases que implica la transferencia tecnológica. A fin de que esta función se realice a cabalidad, se requiere asignar recursos adicionales al propio desarrollo tecnológico para colocar las innovaciones en el mercado.

Tabla 5.

Intervención pedagógica en la función de implantación tecnológica

\begin{tabular}{ll}
\hline Cultura Organizacional & Características \\
\hline $\begin{array}{l}\text { a. Facilitar la instalación de laboratorios de diseño } \\
\text { para el desarrollo de proyectos incluidos en la } \\
\text { cartera tecnológica. }\end{array}$ & $\begin{array}{l}\text { Saber dirigir el proceso creativo en proyectos de diseño } \\
\text { siguiendo las fases de desarrollo, escalamiento e ingeniería. }\end{array}$ \\
$\begin{array}{l}\text { b. Mejorar las condiciones para impulsar el } \\
\text { perfeccionamiento de los procesos de manufactura } \\
\text { en proyectos de diseño. }\end{array}$ & $\begin{array}{l}\text { Saber reconocer las necesidades de escalamiento en la } \\
\text { producción de los proyectos de diseño y dominar el proceso } \\
\text { de manufactura requerido. }\end{array}$ \\
$\begin{array}{l}\text { c. Facilitar a los interesados la amplia disposición de } \\
\text { productos y servicios tecnológicos de calidad. }\end{array}$ & $\begin{array}{l}\text { Saber planificar el esquema de mercadotecnia idóneo a cada } \\
\text { solución de diseño y distinguir el proceso de intervención } \\
\text { que se deba dirigir. }\end{array}$ \\
$\begin{array}{l}\text { d. Flexibilizar los procedimientos en la organización } \\
\text { de grupos interinstitucionales que favorezcan la } \\
\text { realización de proyectos de innovación. }\end{array}$ & $\begin{array}{l}\text { Saber sistematizar los modelos de negociación entre los } \\
\text { grupos de trabajo, a fin de alinear los intereses de las partes } \\
\text { involucradas. }\end{array}$
\end{tabular}

\section{Fuente: elaboración propia}

\section{Deducción cognitiva:}

a. Innovación de productos y servicios. La fase creativa de diseño precisa conducir las iniciativas bajo un riguroso proceso metodológico y bajo un enfoque en particular, según la pauta conceptual que cada protocolo exija. Se completa con la evaluación de los requerimientos observando los parámetros previstos en las alternativas presentadas, en la ingeniería de detalle y, en su caso, en la producción de los modelos y prototipos físicos o digitales. En consecuencia, se ofrece una respuesta relativa a la experiencia de uso y al ciclo de vida del producto.

b. Innovación de procesos de manufactura. Saber detectar y observar las oportunidades para aprovechar el rendimiento de los materiales con base en la transformación más estricta y uniforme al considerar equipos y herramientas de vanguardia. (Hinojosa, 2006). 
c. Innovación de mercadotecnia. Advertir que esos métodos se pueden trabajar, por ejemplo, con nuevos empaques, mejores formas para la distribución y nuevos modelos de promoción del producto, siempre y cuando no afecten los preceptos del desarrollo sustentable (Borja y Ramírez, 2006).

d. Innovación organizacional. Estimular el trabajo multidisciplinar observando las disposiciones normativas e institucionales que regulan a las entidades que participan en los proyectos para acentuar la colaboración dentro y fuera de la organización, a fin de agregar valor a los productos, procesos y métodos que se generen. Compromete decisiones en torno a los modelos de negociación que han de establecerse mediante convenios y contratos que tengan lugar, anticipando el cumplimiento de la voluntad de y entre las entidades vinculantes.

\section{Conclusiones}

A fin de crear experiencias individuales y colectivas que aporten significados diversos en el proceso de aprendizaje, la deducción cognitiva en cada una de las funciones de la innovación tecnológica significa que se puede generar mayor conocimiento a partir de las posibilidades técnicas y científicas que se argumenten en el entorno de la innovación tecnológica. Se entiende que para que en los centros de enseñanza del diseño se logre ese cometido se requiere seguir tantas prácticas como sea necesario para involucrar los intereses y los conocimientos de los profesionales, los usuarios, los empresarios o los financiadores de proyectos específicos. La academia de diseño debe permanecer vigilante al llamado de prácticas disruptivas para formar talentos desde una apuesta multidimensional.

La dirección de proyectos de innovación, desde la práctica académica, reclama nuevos esquemas programáticos y pedagógicos que se fundamenten desde el campo de la gestión de tecnología, toda vez que es un buen referente que aporta validez a los desarrollos y permite medir la competitividad de los emprendedores. Es una oportunidad que confluye en un cúmulo de posibilidades didácticas que abordan el pensamiento complejo; sin embargo, el reto radica en saber convertir el intelecto y el proceso creativo en una amplia cartera de propuestas que resulten en activos tangibles e intangibles que tengan viabilidad de ser transferidos con éxito en cualquiera de los sectores socio-productivos.

La práctica que tiene que ver con dicho cometido habla de una organización flexible con miras a experimentar ciertas metodologías hasta ahora inexploradas, desde las cuales se pueden originan los productos, los procesos y los métodos que, una vez que las experiencias de los usuarios muestran su satisfacción, adquieren valor.

Las competencias en la dirección y en la didáctica de los proyectos de innovación se adquieren una vez que se ponen en marcha las prácticas de colaboración multidimensionales, en donde la mediación pedagógica debe ser muy puntual al provocar una interacción cognitiva permanente con especialistas para desarrollar cada una de las funciones del modelo de gestión de tecnología. El reto está en adaptarse a esta otra cultura de trabajo de manera activa, reflexiva y crítica bajo estrictos parámetros de reciprocidad para aprender el ciclo completo de la innovación en proyectos de diseño, lo cual hace oportuna la propuesta de crear un espacio dedicado, no sólo a incrementar el conocimiento en esta materia, sino a 
reorientar el ya existente hacia la satisfacción de nuevas necesidades para equilibrar las oportunidades, que desde el diseño se pueden gestionar para ofrecer soluciones a una gran variedad de problemas a los que las futuras generaciones se enfrentarán.

\section{Bibliografía}

Álvarez, M. M. (enero-junio 2011). Perfil del docente en el enfoque basado en competencias. Revista Electrónica Educare, XV (1), p. 99-107 Universidad Nacional Heredia, Costa Rica. Recuperado de: http://www.redalyc.org/articulo.oa?id=194118804008

Barjau, E. (2006). Planeación de Tecnología. Cuadernos de gestión de tecnología. Ciudad de México, México: Ed. Fundación del Premio Nacional de Tecnología.

Borja Ramírez, V., y Ramírez Reivich, A. C. (2006). Innovación de producto. Cuadernos de gestión de Tecnología. Ciudad de México, México: Ed. Fundación del Premio Nacional de Tecnología.

Cámara de Diputados del H. Congreso de la Unión. (Última reforma publicada el 18-05-2018). Ley de la Propiedad Industrial. Estados Unidos Mexicanos. Recuperado de: http://www.diputados.gob.mx/LeyesBiblio/pdf/50_180518.pdf

Campos, Vergara, F., Bolbarán, Ramírez, J., Bustos, Raggi, C., y González, Vallejos, M. (2014). Formación de directores de excelencia: un mismo objetivo distintas demandas. Perspectiva educacional. Formación de profesores, 53(2), 91-111.

Díaz, Palacios, J. A. (2013). Calidad Educativa: Un análisis sobre la acomodación de los sistemas de gestión de la calidad empresarial a la valoración en educación. Tendencias Pedagógicas, 21, 177-194.

FPNTI-Fundación Premio Nacional de Tecnología e Innovación, A.C. (2016). Modelo Nacional de Gestión de la Tecnología. Creando entornos que generan innovación. (XVIII Edición). Ciudad de México, México: Recuperado de: http://www.fpnt.org.mx/images/stories/Calendario2015/MODELO-GDT.pdf

Hernández, R. G. (2008). Los constructivismos y sus implicaciones para la educación. Perfiles Educativos, XXX (122), 38-77. Universidad Nacional Autónoma de México, Instituto de Investigaciones sobre la Universidad y la Educación.

Hinojosa, M. (2006). Innovación de proceso. Cuadernos de gestión de tecnología. Ciudad de México, México: Ed. Fundación del Premio Nacional de Tecnología.

IMNC-Instituto Mexicano de Normalización y Certificación, A.C. (2007a). Sistema de Gestión de la Tecnología - Terminología. Norma Mexicana NMX-GT-001-IMNC. Estados Unidos Mexicanos: Autor.

IMNC-Instituto Mexicano de Normalización y Certificación, A.C. (2007b). Sistema de Gestión de la Tecnología - Requisitos. Norma Mexicana NMX-GT-001-IMNC. Estados Unidos Mexicanos: Autor

Morín, E. (1999). Los siete saberes necesarios para la educación del futuro. Organización de las Naciones Unidas, Francia: UNESCO. Traducción Mercedes Vallejo-Gómez, Profesora de la UPB - Medellín, Colombia, con la colaboración de Nelson Vallejo-Gómez y Françoise Girard.

OMPI (2019). Glosario de los términos más importantes relacionados con la propiedad intelectual y los recursos genéticos, los conocimientos tradicionales y las expresiones culturales tradicionales. Ginebra, Suiza. Recuperado de: https://www.wipo.int/edocs/mdocs/tk/es/wipo_grtkf_ic_40/wipo_grtkf_ic_40_inf_7.pdf 
OEI-Organización de los Estados Iberoamericanos para la Educación, la Ciencia y la Cultura. (2014). Informe sobre Tendencias Sociales y Educativas en América Latina. España: Autor.

Organización para la Cooperación y el Desarrollo Económicos. (2003). Manual de Frascati. Propuesta de norma práctica para encuestas de investigación y desarrollo experimental. España: Fundación Española Ciencia y Tecnología. 